NANOSYSTEMS: PHYSICS, CHEMISTRY, MATHEMATICS, 2015, 6 (2), P. 224-243

\title{
Time-dependent quantum circular billiard
}

\author{
${ }^{1,2}$ D. B. Babajanov, ${ }^{1,2}$ D. U. Matrasulov, ${ }^{1,3}$ Z. A. Sobirov, ${ }^{4}$ S. K. Avazbaev, ${ }^{1,2}$ O. V. Karpova \\ ${ }^{1}$ National University of Uzbekistan, Tashkent, Uzbekistan \\ 2 Turin Polytechnic University in Tashkent, 17. Niyazov Str., 100095, Tashkent, Uzbekistan \\ 3 Tashkent Financial Institute, 60A, Amir Temur Str., 100000 Tashkent, Uzbekistan \\ 4 ARC Centre for Antimatter-Matter Studies, Department of Applied Physics, Curtin \\ University, G.P.O. Box U1987, Perth 6845, Australia \\ *d_babajanov@yahoo.com
}

\section{PACS 03.65.Ge}

DOI 10.17586/2220-8054-2015-6-2-224-243

The motion of a quantum particle in a time-dependent circular billiard is studied on the basis of the Schrödinger equation with time-dependent boundary conditions. The cases of monotonically expanding (contracting), non-harmonically, harmonically breathing circles the case when billiard wall suddenly disappears are explored in detail. The exact analytical solutions for monotonically expanding and contracting circles are obtained. For all cases, the time-dependence of the quantum average energy is calculated. It is found that for an harmonically breathing circle, the average energy is time-periodic in the adiabatic regime with the same period as that of the oscillation. For intermediate frequencies which are comparable with the initial frequency of the particle in unperturbed billiard, such periodicity is broken. However, for very high frequencies, the average energy once again becomes periodic. A qualitative analysis of the border between adiabatic and non-adiabatic regimes is provided.

Keywords: quantum billiards, Schrödinger equation.

Received: 2 February 2015

\section{Introduction}

Billiards are convenient models for the study of classical and quantum dynamics of non-integrable systems. They have been extensively studied in both experimental [1] and theoretical contexts [2,3]. A remarkable feature of particle motion in billiards is the dependence of the dynamics on the geometry of the billiard boundaries. Depending on the geometry, the dynamics can be regular, mixed or chaotic in the classical case. The corresponding quantum dynamics exhibit certain features in the statistical properties of the energy spectrum. For those systems, whose classical dynamics are chaotic, the nearest-neighbor energy level spacing distribution function of the corresponding quantum system is of Wigner type, while for regular systems this distribution is Poissonian [2,3]. Most of the studies on billiards deal with static billiards. However, in recent years there is a growing interest in the classical dynamics of time-dependent billiards [4]- [8]. One of the key questions that has been investigated is whether there will be Fermi acceleration in such two-dimensionally confined geometries. Studies of time-dependent billiards with a regular static counterpart, such as the breathing circle, show that there is no unbounded growth of the velocity, while for some non-integrable geometries, like the stadium [4,5] and the (eccentric) annular billiard [6], Fermi acceleration is possible. Recently, the classical dynamics of particles in time-dependent elliptic billiard have been studied and tunable Fermi acceleration has been shown to exist in such a system, even though the static counterpart is integrable [7,8]. Despite certain progress made in the study of the classical dynamics of time-dependent billiards, the quantum dynamics of such systems are still an open problem. At the present time, no detailed treatment of the problem 
on the basis of two-dimensional time-dependent Schrödinger equation has yet been done. In the quantum case, the problem of time-dependence is reduced to solving the two-dimensional Schrödinger equation with time-dependent boundary conditions. The one-dimensional counterpart of this problem has been extensively studied [10] - [28]. These studies showed that even for the one-dimensional case, the Schrödinger equation with time-dependent boundary conditions cannot be solved exactly for an arbitrary time-dependence of the boundary conditions. In the case of the 1D box, the time-dependent boundary conditions can be reduced to static ones, leading to a Schrödinger equation which can be interpreted as a time-dependent confined harmonic oscillator [13,16-18]. Makowsky et al. solved this problem for special cases of the time-dependence of the boundaries [16]. In particular, they classified the types of time-dependent boundary conditions for which an exact analytical solution of the problem can be obtained.

In the case of periodic (harmonic) time-dependence of the boundaries, the problem can be solved numerically by an expansion of the wave function in terms of Gaussian wave packets [17]. Scheininger and Kleber treated the case of a special type of periodically timedependent boundaries by solving the problem in terms of the full-circle propagator [15]. Seba studied the case of time-periodic boundary conditions in terms of Floquet operators [24]. In a very recent work, Ref. [28], the quantum infinite square well with an oscillating wall was studied. It was shown that three types of regimes are possible in such a system, which can be classified as adiabatic (for low oscillation frequencies), chaotic (for intermediate frequencies) and periodic (for high oscillation frequencies). In particular, the average energy was found to be time-periodic for the adiabatic and periodic regimes, while for the chaotic regime, periodicity was broken.

In this work, we address the two-dimensional extension of the problem considered by Makowski et al. [16]. We solve the Schrödinger equation for the circular billiard with a time-dependent radius. In particular, we consider the following cases:

i) monotonically expanding (contracting) circle;

ii) non-harmonically breathing circle;

iii) harmonically breathing circle.

The classical counterpart of this system has been studied earlier Ref. [30], where it was shown that unbounded velocity gain is not possible. In Ref. [31] dynamics, statistical properties of quasi-energy levels and wave functions for the quantum system have been studied for harmonically oscillating circle. The "scars" in the quantum quasi-energy eigenfunctions corresponding to classical unstable periodic orbits were found in [31].

This paper is organized as follows: in the next section we briefly recall the case of the circular billiard with fixed boundaries. Section 3 presents the analytical solution when time and coordinate variables can be separated. In section 4, we study the case when billiard wall disappears suddenly. Non-harmonically breathing circle is studied in section 5. Section 6 provides detailed study of the circle with harmonically oscillating boundaries, by solving the time-dependent Schrödinger equation numerically. Finally, section 7 presents the discussion of the obtained results and some concluding remarks.

\section{Static circular billiard}

The circular billiard is defined by the potential:

$$
V(r, \theta)=V(r)=\left\{\begin{array}{c}
0 \text { for } r<r_{0}, \\
\infty \text { for } r \geqslant r_{0}
\end{array}\right.
$$


where $r_{0}$ is the radius of circle. The corresponding quantum mechanical eigenvalue problem is given by:

$$
-\frac{\hbar^{2}}{2 \mu}\left(\frac{\partial^{2}}{\partial r^{2}}+\frac{1}{r} \frac{\partial}{\partial r}+\frac{1}{r^{2}} \frac{\partial^{2}}{\partial \theta^{2}}\right) \psi(r, \theta)=E \psi(r, \theta),
$$

with $\mu$ being the mass of the particle. Angular and radial variables in this equation can be separated with $\psi(r, \theta)=R(r) \Theta(\theta)$ (the system of units $\mu=\hbar=1$ is used throughout the paper)

$$
\begin{gathered}
-\frac{1}{2}\left(\frac{d^{2} R}{d r^{2}}+\frac{1}{r} \frac{d R}{d r}\right)+\frac{m^{2}}{2 r^{2}} R=E R, \\
\frac{d^{2} \Theta_{(m)}(\theta)}{d \theta^{2}}=-m^{2} \Theta_{(m)}(\theta),
\end{gathered}
$$

where $R(r)$ and $\Theta(\theta)$ are the radial and angular solutions, respectively, and $m$ is the angular quantum number. The boundary conditions for Eqs. 3 and 4 are given by:

$$
\begin{gathered}
R\left(r_{0}\right)=0 \\
\Theta_{(m)}(\theta+2 \pi)=\Theta_{(m)}(\theta) .
\end{gathered}
$$

We note that using the substitution $R(r)=u(r) / \sqrt{r}$, Eq.(3) can be reduced into a form which does not contain the first derivative of the radial wave function:

$$
-\frac{1}{2} \frac{d^{2} u}{d r^{2}}+\frac{m^{2}-1 / 4}{2 r^{2}} u=E u
$$

The solution for the radial equation satisfying the above boundary condition can be written in terms of the regular Bessel functions [29,33-35]:

$$
R_{m n}(r)=N_{m n} J_{m}\left(\lambda_{m n} r\right)
$$

where $n$ is the radial quantum number and the eigenvalues are defined by:

$$
E_{m n}=\frac{\lambda_{m n}^{2}}{2 r_{0}^{2}}
$$

$\lambda_{m n}$ is the $n$-th zero of the $m$-th Bessel function $J_{m}(r)$ and $N_{m n}$ is the normalization constant given by:

$$
N_{m n}^{2} \int_{0}^{r_{0}}\left|J_{m}\left(\lambda_{m n} r\right)\right|^{2} r d r=1 .
$$

The angular equation has normalized solutions of the form:

$$
\Theta_{(m)}(\theta)=\frac{1}{\sqrt{2 \pi}} e^{i m \theta}
$$

Eqs. (6) -(9) completely define the solution of the time-independent quantum mechanical circular billiard problem. 


\section{Monotonically expanding circle}

Now, we proceed to a time-dependent circle. The central symmetry of the circular billiard allows us to solve the problem within the radial Schrödinger equation. We restrict ourselves to the case when just the radius of the circle is time-dependent so that central symmetry is remained. First, we consider a monotonically expanding (contracting) circle. For circular billiards with time-dependent radius, the radial Schrödinger equation can be written as:

$$
i \frac{\partial R}{\partial t}=-\frac{1}{2} \frac{\partial^{2} R}{\partial r^{2}}-\frac{1}{2 r} \frac{\partial R}{\partial r}+\frac{m^{2}}{2 r^{2}} R
$$

and the boundary conditions are given by:

$$
R(r(t), t)=0 \text { for } r=r_{0}(t)
$$

As shown earlier for example in Refs. [11]- [18], to solve Eq.(10) we need to make the boundary conditions time-independent. This is can done by using the coordinate transformation:

$$
y=\frac{r}{r_{0}(t)}
$$

Thus, Eq.(10) reduces to:

$$
i \frac{\partial R(y, t)}{\partial t}=-\frac{1}{2 r_{0}^{2}} \frac{\partial^{2} R}{\partial y^{2}}-\left(\frac{1}{2 r_{0}^{2} y}-i \frac{\dot{r}_{0}}{r_{0}} y\right) \frac{\partial R}{\partial y}+\frac{m^{2}}{2 r_{0}^{2} y^{2}} R .
$$

Inserting the substitution:

$$
R(y, t)=\frac{1}{r_{0}(t) \sqrt{y}} \exp \left(\frac{i}{2} \dot{r}_{0}(t) r_{0}(t) y^{2}\right) \phi(y, t),
$$

into Eq.(12), we get:

$$
i r_{0}^{2} \frac{\partial \phi}{\partial t}=-\frac{1}{2} \frac{\partial^{2} \phi}{\partial y^{2}}+\left(\frac{1}{2} r_{0}^{3} \ddot{r}_{0} y^{2}+\frac{m^{2}-1 / 4}{2 y^{2}}\right) \phi .
$$

Eq.(14) can be interpreted as the Schrödinger equation for a time-dependent harmonic oscillator confined to a unit circle. Time and coordinate variables in Eq.(14) can be separated, provided that the following condition is fulfilled:

$$
r_{0}^{3} \ddot{r}_{0}=\text { const }=-C, C>0 .
$$

Separating variables by:

$$
\phi(y, t)=\varphi(y) T(t)
$$

we get:

$$
-\frac{1}{2} \frac{d^{2} \varphi}{d y^{2}}+\left(-\frac{1}{2} C y^{2}+\frac{m^{2}-1 / 4}{2 y^{2}}\right) \varphi=k^{2} \varphi
$$

and

$$
i r_{0}^{2} \frac{d T}{d t}=k^{2} T(t)
$$

where $k$ is the separation constant. The general solution for Eq.(15) can be written as:

$$
r_{0}(t)=\sqrt{a t^{2}+b t+c}
$$

where

$$
C=\frac{b^{2}-4 a c}{4}
$$


For $C=\frac{b^{2}}{4}$ we have a circle expanding with non-constant velocity:

$$
r_{0}(t)=\sqrt{a t+b}
$$

For $C=0$ this solution corresponds to a linearly expanding (contracting) circle:

$$
r_{0}(t)=a t+b \text {. }
$$

For the linearly expanding (contracting) circle, Eq. (16) is formally the same as Eq. (5), the one for the static circular billiard. However, because of the relation (13), the solution of the original time-dependent radial equation (10) is different from that of the static circular billiard. For the time-dependent radial wave function, we have:

$$
R_{m n}(r, t)=\frac{1}{r_{0}(t) J_{m+1}\left(\lambda_{m n}\right)} \exp \left(\frac{i}{2} \frac{\dot{r}_{0}(t)}{r_{0}(t)} r^{2}\right) J_{m}\left(\frac{\lambda_{m n} r}{r_{0}(t)}\right) \exp \left(-i \int_{0}^{t} \frac{\lambda_{m n}^{2} d \tau}{r_{0}^{2}(\tau)}\right)
$$

For $a=0$, the solution coincides with the one of the static billiard given by Eq.(6). For $C \neq 0$, the solution of Eq. (16) is expressed in terms of the confluent hypergeometric functions [35]:

$$
\varphi(y)=y^{m+1 / 2} e^{-0.5 i \sqrt{C} y^{2}} M\left(d, m+1, i \sqrt{C} y^{2}\right),
$$

where:

$$
d=\frac{m+1}{2}+\frac{k^{2}}{2 \sqrt{C}} i
$$

The eigenvalues $k$ are defined by the condition $\varphi(1)=0$, or:

$$
M(d, m+1, i \sqrt{C})=0,
$$

where only $d$ depends on $k$. Thus the solution of Eq.(10) can be written as:

$$
\begin{gathered}
R_{m n}(r, t)=N_{m n} \frac{r^{m}}{r_{0}^{m+1}(t)} \exp \left(\frac{i r^{2}}{2}\left(\frac{\dot{r}_{0}(t)}{r_{0}(t)}-\frac{\sqrt{C}}{r_{0}^{2}(t)}\right)\right) \times \\
\times M\left(\frac{m+1}{2}+\frac{k_{m n}^{2}}{2 \sqrt{C}} i, m+1, i \sqrt{C} \frac{r^{2}}{r_{0}^{2}(t)}\right) \exp \left(-i k_{m n}^{2} \int_{0}^{t} \frac{d \tau}{r_{0}^{2}(\tau)}\right),
\end{gathered}
$$

where $N_{m n}$ is the normalization constant given by

$$
\int\left|R_{m n}\right|^{2} r d r=1
$$

A quantity which is of interest from the viewpoint of Fermi acceleration in timedependent billiards, is the average kinetic energy, which is defined as:

$$
\left\langle E_{m}(t)\right\rangle=\langle\psi(r, t)|H| \psi(r, t)\rangle
$$

where:

$$
H=-\frac{1}{2} \frac{\partial^{2}}{\partial r^{2}}-\frac{1}{2 r} \frac{\partial}{\partial r}+\frac{m^{2}}{2 r^{2}}
$$

The asymptotic behavior of the average energy for the linearly expanding (contracting) circle in the limits $t \rightarrow \infty$ and $r_{0}(t) \rightarrow \infty$ can easily be estimated. Indeed, since radial and angular variables are separated and $H$ does not depend on the angular variables, the average energy can be written in terms of the time-dependent radial wave functions as:

$$
\left\langle E_{m n}(t)\right\rangle=\int_{0}^{r_{0}(t)} R_{m n}^{*}(r, t) H R_{m n}(r, t) r d r=\frac{1}{2} \int_{0}^{r_{0}(t)}\left|\frac{\partial R_{m n}}{\partial r}\right|^{2} r d r+\frac{m^{2}}{2} \int_{0}^{r_{0}(t)} \frac{\left|R_{m n}\right|^{2}}{r} d r .
$$


Taking into account the relation:

$$
\left|\frac{\partial R_{m n}}{\partial r}\right|^{2}=\frac{1}{r_{0}^{2}(t) J_{m+1}^{2}\left(\lambda_{m n}\right)}\left[\left(\frac{\dot{r_{0}}}{r_{0}}\right)^{2} J_{m}^{2}\left(\frac{\lambda_{m n} r}{r_{0}}\right) r^{2}+\left(\frac{\partial}{\partial r} J_{m}\left(\frac{\lambda_{m n} r}{r_{0}}\right)\right)^{2}\right],
$$

we get:

$$
\left\langle E_{m n}(t)\right\rangle=C_{1}+C_{2} \frac{1}{(a t+b)^{2}},
$$

where the constants $C_{1}$ and $C_{2}$ are given by:

$$
\begin{gathered}
C_{1}=\frac{a^{2}}{J_{m+1}^{2}\left(\lambda_{m n}\right)} \int_{0}^{1} y^{3} J_{m}^{3}\left(\lambda_{m n} y\right) d y \\
C_{2}=\frac{1}{J_{m+1}^{2}\left(\lambda_{m n}\right)} \int_{0}^{1}\left(\frac{\partial}{\partial y} J_{m}\left(\lambda_{m n} y\right)\right)^{2} y d y+\frac{m^{2}}{J_{m+1}^{2}\left(\lambda_{m n}\right)} \int_{0}^{1} \frac{1}{y} J_{m}^{2}\left(\lambda_{m n} y\right) d y .
\end{gathered}
$$

From Eq.(26), the average energy of the particle in a linearly expanding $(a>0)$ circle goes to $E_{m n}(t) \rightarrow C_{1}$ for $t \rightarrow \infty$, while for the linearly contracting $(a<0, b>0)$ circle we get asymptotically $E_{m n}(t) \rightarrow+\infty$ for $t \rightarrow-\frac{b}{a}$. Such asymptotic behavior is confirmed by Fig. 1, where $\left\langle E_{m n}(t)\right\rangle$ is shown for the linearly expanding and contracting circles for different values of the expanding (contracting) velocities $a$. It is clear that the difference between adiabatic $(a<<1)$ and non-adiabatic $(a>>1)$ regimes is exhibited in the decay rate of the curve. Furthermore, the curves are symmetric for the expanding and contracting circles (for the same expanding/contracting velocities). We note that the above described asymptotic behavior is true not only for linearly expanding (contracting) circles but also for other types of monotonically expanding circles, as long as the radius is given by either Eqs. (18) or (19).

\section{Suddenly removed billiard walls}

In the time-dependent billiard problem, it is important to explore differences between adiabatic and non-adiabatic regimes of the wall's motion. In this section, we consider the case when the billiard wall is removed (disappears) suddenly. To some extent, this situation is equivalent to when the billiard expands with infinite velocity, so that it can be considered a highly non-adiabatic regime. A similar problem for the one-dimensional infinite well has been previously considered [27] in the context of diffraction in time. Our purpose is to explore the time-evolution of the wavefunction, mean position, and its time derivative for such regime of wall's motion. We assume that at $t=0$ the initial state wavefunction of the system is the eigenstate of the circular billiard given by:

$$
\varphi_{m n}(r, \theta)=\frac{\sqrt{2}}{r_{0} J_{m+1}\left(\lambda_{m n}\right)} J_{m}\left(\frac{\lambda_{m n} r}{r_{0}}\right) e^{i m \theta} .
$$

Time evolution of the wavefunction can be calculated using the Green's function, $G\left(\mathbf{r}, t ; \mathbf{r}^{\prime}, 0\right)$ $[27]$

$$
\psi(\mathbf{r}, t)=\int d \mathbf{r}^{\prime} G\left(\mathbf{r}, t ; \mathbf{r}^{\prime}, 0\right) \psi\left(\mathbf{r}^{\prime}, 0\right)
$$

For the case of circular billiard the time evolution is given by [polyanin]

$$
\psi(r, \theta, t)=\frac{1}{2 \pi i t} \int_{0}^{2 \pi} \int_{0}^{r_{0}} r^{\prime} \exp \left[i \frac{r^{2}+r^{\prime 2}-2 r r^{\prime} \cos \left(\theta-\theta^{\prime}\right)}{2 t}\right] \varphi\left(r^{\prime}, \theta^{\prime}\right) d r^{\prime} d \theta^{\prime},
$$



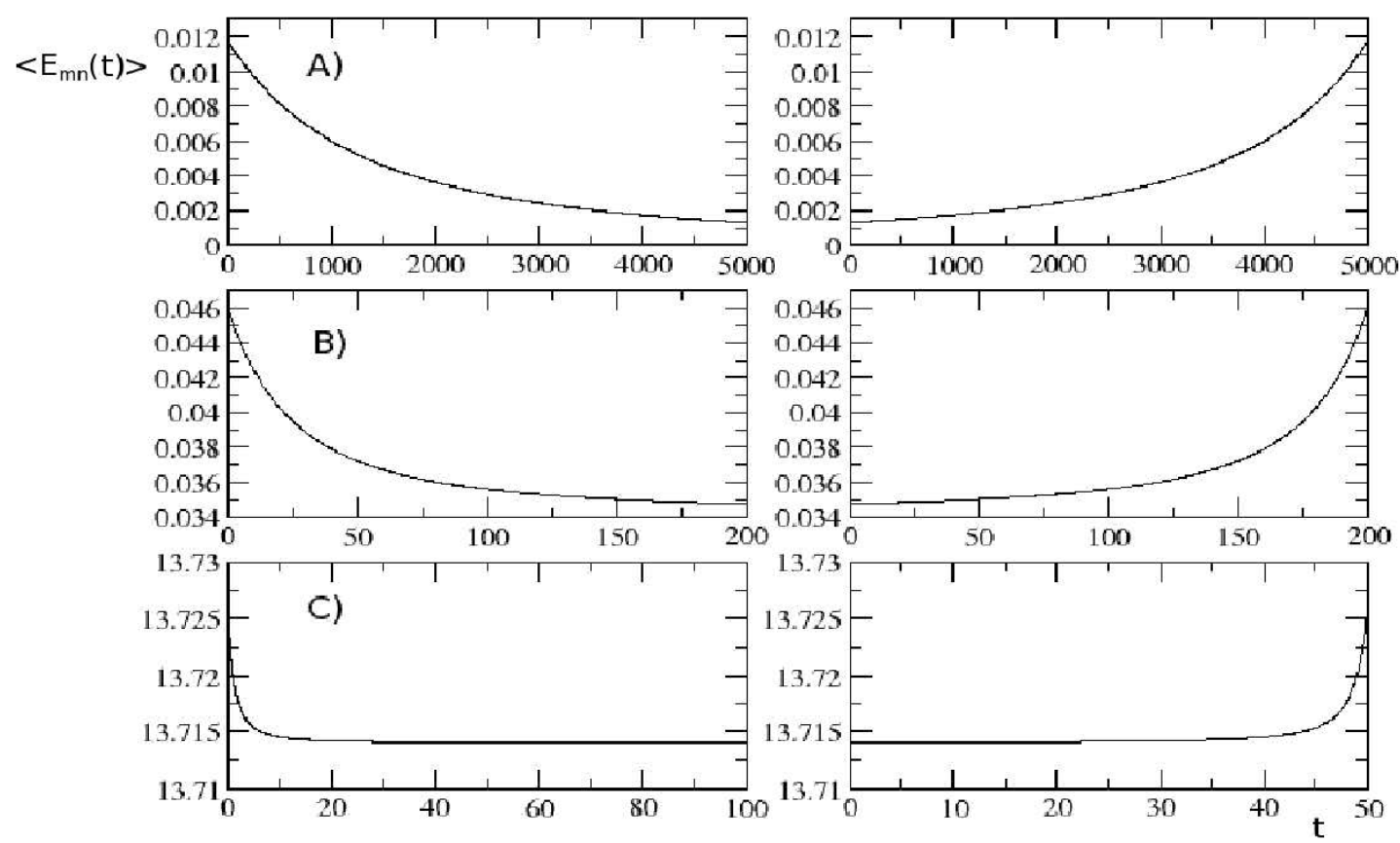

FIG. 1. The time-dependence of the quantum average energy for linearly expanding $r_{0}(t)=b_{1}+a t$ (left panel) and (with the same velocity as that of the expanding circle) contracting $r_{0}(t)=b_{2}-a t$ (right panel) circles. The initial state is taken to be an eigenstate of (16) with $m=1, n=1$. The parameters are: A) $\left.\left.a=0.01, b_{1}=25, b_{2}=75, \mathrm{~B}\right) a=0.5, b_{1}=25, b_{2}=125, \mathrm{C}\right)$ $a=10.0, b_{1}=25, b_{2}=525$

and for $m=0$ :

$$
\psi(r, t)=\frac{1}{i t} \int_{0}^{r_{0}} r^{\prime} \exp \left[i \frac{r^{2}+r^{\prime 2}}{2 t}\right] I_{0}\left(-\frac{i r r^{\prime}}{t}\right) \varphi\left(r^{\prime}\right) d r^{\prime},
$$

where $I_{0}\left(r^{\prime}\right)$ is the modified Bessel function. The wavefunction is normalized as:

$$
N(t)=\int_{0}^{\infty}|\psi|^{2} r d r=1
$$

The mean position can be calculated as:

$$
\langle r(t)\rangle=\int_{0}^{\infty}|\psi|^{2} r^{2} d r
$$

In Fig. 2, we show the $r$-dependence of the $m=0, n=3$ circular billiard wavefunction (with initial radius $\left.r_{0}=40\right)$ at different moments of time $(t=100, t=300, t=500)$. It is clear that the wavefunction decays with increasing $r$ and completely disappears at upper $r$ limit. The decay distance is longer as $t$ is longer. In addition, we explored the time evolution of the wavefunction, mean position and its time derivative after removing the wall. As an initial state, we chose the static billiard wavefunction for $m=0,1,2, n=1,2,3$. The results are shown in Fig. 3. As we can see in Fig 3, where the mean position (left panel) and its time derivative (right panel), after some initial period the states will expand with constant velocity. As can be seen from Fig. 3, $\langle r(t)\rangle$ grows monotonically in time. This implies that the motion of the particle is not localized after the wall's removal and can go to infinity; as large as the initial state energy and as high as the growth rate of $\langle r\rangle$. Completely different 
behavior can be observed for $\frac{d\langle r(t)\rangle}{d t}$; unlike the mean position, $\frac{d\langle r(t)\rangle}{d t}$ grows during some initial time period, after which it becomes constant. Again, the growth rate is proportional to the initial state energy. The accuracy of wavefunction evolution has been checked by monitoring norm conservation.

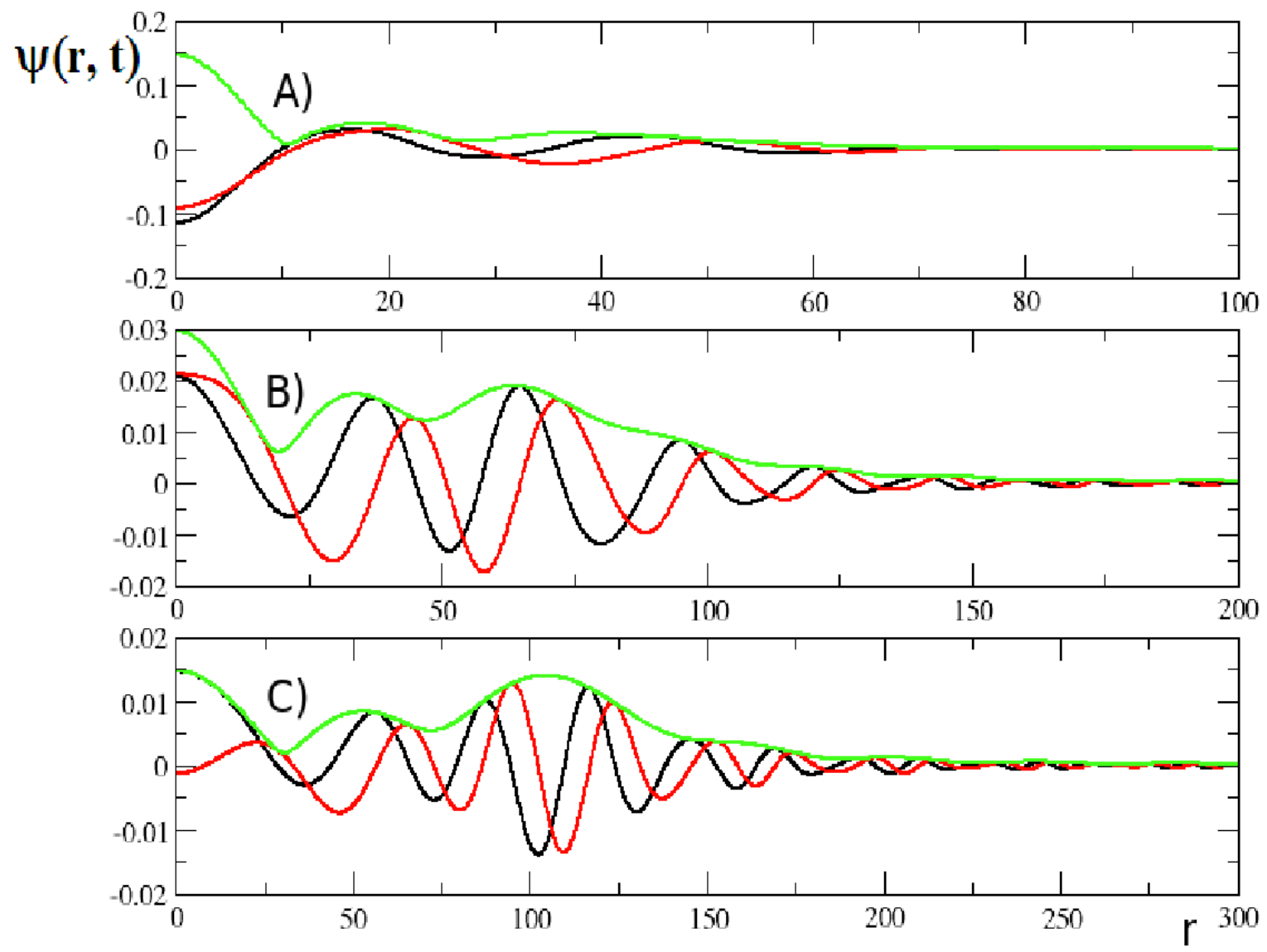

FIG. 2. The wavefunction of $m=0, n=3$ state as a function of $r$ (the real part (black), the imaginary part (red), the absolute value of the wavefunction (green)): A) $t=100, \mathrm{~B}) t=300$, C) $t=500$

\section{Non-harmonically breathing circle}

In this section, we consider the following type of time- dependence:

$$
r_{0}(t)=\left\{\begin{array}{c}
\rho_{0}+v t, \quad 0<t<\frac{1}{2} T, \\
\rho_{0}+v(T-t), \quad \frac{1}{2} T<t<T .
\end{array}\right.
$$

This means that the time-law of the radius is periodic but not harmonic and it is still possible to find an exact solution of Eq.(14). For a fixed value of $T$, it is clear that the oscillation amplitude depends on the velocity, $v$ : the higher the velocity the larger the amplitude will be. The oscillation frequency is defined as $\omega=2 \pi / T$. We have to solve Eq.(14) with the boundary conditions $\phi(0, t)=\phi(1, t)=0$. The boundary condition at $y=0$ follows from the substitution (13). It is clear that the motion of the boundary can be considered as the (subsequent) combination of linearly expanding and contracting circles. 

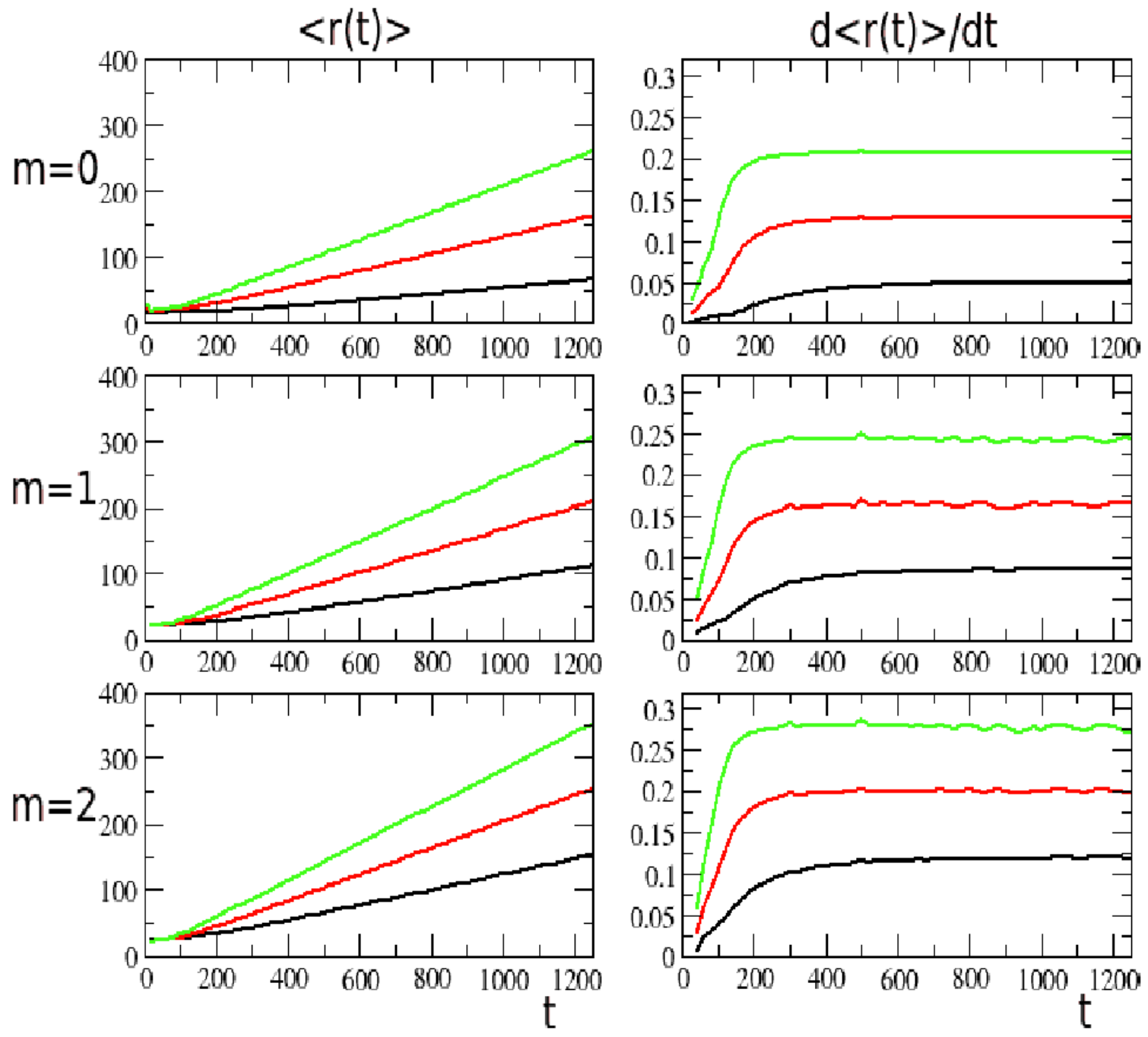

FIG. 3. Mean position and its time-derivative (expansion rate). $n=1$ (black), $n=2$ (red), $n=3$ (green)

The one-dimensional Schrödinger equation with such a boundary condition is solved in Ref. [15] in terms of the full-cycle propagator. Here, we use the same prescription as in Ref. [15] to obtain the solution for Eq.(14). The solution for Eq.(14) can be found in each time interval, $0<t<\frac{1}{2} T$ and $T<t<T$, from which, general solutions can be constructed. First, we note that for $r_{0}^{3} \ddot{r}_{0}=0$, time and coordinate variables in Eq.(14) can be separated and the solution can be written as:

$$
\phi_{m n}(y, t)=\frac{\sqrt{2}}{J_{m+1}\left(\lambda_{m n}\right)} \sqrt{y} J_{m}\left(\lambda_{m n} y\right) \exp \left(-2 i \lambda_{m n}^{2} \int_{0}^{t} \frac{d \tau}{r_{0}^{2}(\tau)}\right)
$$

where $0<\lambda_{m 1},<\lambda_{m 2},<\ldots$ are zeros of the Bessel function given by $J_{m}\left(\lambda_{m n}\right)=0$. In the following, the angular quantum number $m$ will be considered as fixed, and consequently, we will omit the subscript $m$ in the following. The solution of Eq.(14) in the time interval 
$0<t<\frac{1}{2} T$ can be written in terms of the functions (36) as:

$$
\phi(y, t)=\sum_{n=1}^{\infty} A_{n} \phi_{n}(y, t) .
$$

The expansion coefficients can be found as Fourier coefficients using the initial value of $\phi$ i.e. from the relation:

$$
\phi(y,+0)=\sum_{n=1}^{N} A_{n} Y_{n}(y)
$$

that gives:

$$
A_{n}=\int_{0}^{1} Y_{n}(y) \cdot \phi(y,+0) d y
$$

where:

$$
Y_{n}(y)=\frac{\sqrt{2}}{J_{m+1}\left(\lambda_{m n} y\right)} \sqrt{y} J_{m}\left(\lambda_{m n} y\right) .
$$

To find the solution in the general case, we note that:

$$
r_{0} \ddot{r}_{0} y^{2}=-2 v y^{2}\left[\rho_{1} \delta\left(t-\frac{1}{2} T\right)-\rho_{0} \delta(t-T)+\ldots\right],
$$

where $\rho_{1}=\rho_{0}+\frac{1}{2} v T$. The solution of Eq.(14) jumps at $t=\frac{1}{2} T$ :

$$
\phi\left(y, \frac{1}{2} T+0\right)=\exp \left(i v y^{2} \rho_{1}\right) \phi\left(y, \frac{1}{2} T-0\right) .
$$

The solution of Eq.(14) in the interval $\frac{1}{2} T<t<T$ can be written as:

$$
\phi(y, t)=\sum_{n=1}^{\infty} \tilde{A}_{n} Y_{n}(y) \exp \left(-2 i \lambda_{m n}^{2} \int_{T / 2}^{t} \frac{d \tau}{r_{0}^{2}(\tau)}\right),
$$

where Fourier coefficients can be found as:

$$
\begin{aligned}
\tilde{A}_{n}= & \int_{0}^{1} Y_{n}(y) \phi\left(y, \frac{1}{2} T+0\right) d y=\int_{0}^{1} Y_{n}(y) e^{i v y^{2} \rho_{1}} \phi\left(y, \frac{1}{2} T-0\right) d y= \\
& =\sum_{l} A_{l} \exp \left(-2 i \lambda_{m l}^{2} \int_{0}^{T / 2} \frac{d \tau}{r_{0}^{2}(\tau)}\right) \int_{0}^{1} Y_{n}(y) e^{i v y^{2} \rho_{1}} Y_{l}(y) d y .
\end{aligned}
$$

At $t=T$ the solution has a jump:

$$
\phi(y, T+0)=e^{-i v y^{2} \rho_{0}} \phi(y, T-0) .
$$

Expanding $\phi(y, T+0)$ in terms of $\phi_{n}(y, T+0)$, we get:

$$
\phi(y, T+0)=\sum_{n} \bar{A}_{n} \phi(y,+0)=\sum_{n} \bar{A}_{n} Y_{n}(y),
$$

we can find expansion coefficients as:

$$
\bar{A}_{n}=\int_{0}^{1} Y_{n}(y) \phi(y, T+0) d y=\int_{0}^{1} Y_{n}(y) e^{-i v \rho_{0}} \phi(y, T-0) d y=
$$




$$
=\sum_{j} \tilde{A}_{j} \exp \left(-2 i \lambda_{m j}^{2} \int_{T / 2}^{T} \frac{d \tau}{r_{0}^{2}(\tau)}\right) \int_{0}^{1} Y_{n}(y) e^{i v y^{2} \rho_{1}} Y_{j}(y) d y=\sum_{l} A_{l} U_{n l} .
$$

The full-cycle propagator is given by:

$$
U_{n l}=\sum_{j} C_{j l}\left(\rho_{1}\right) \exp \left(-i \frac{T}{\rho_{0} \rho_{1}} \lambda_{m l}^{2}\right) C_{n j}^{*}\left(\rho_{0}\right) \exp \left(-i \frac{T}{\rho_{0} \rho_{1}} \lambda_{m j}^{2}\right),
$$

with

$$
C_{n j}(\rho)=\int_{0}^{1} Y_{n}(y) e^{i v y^{2} \rho} Y_{j}(y) d y
$$

A)
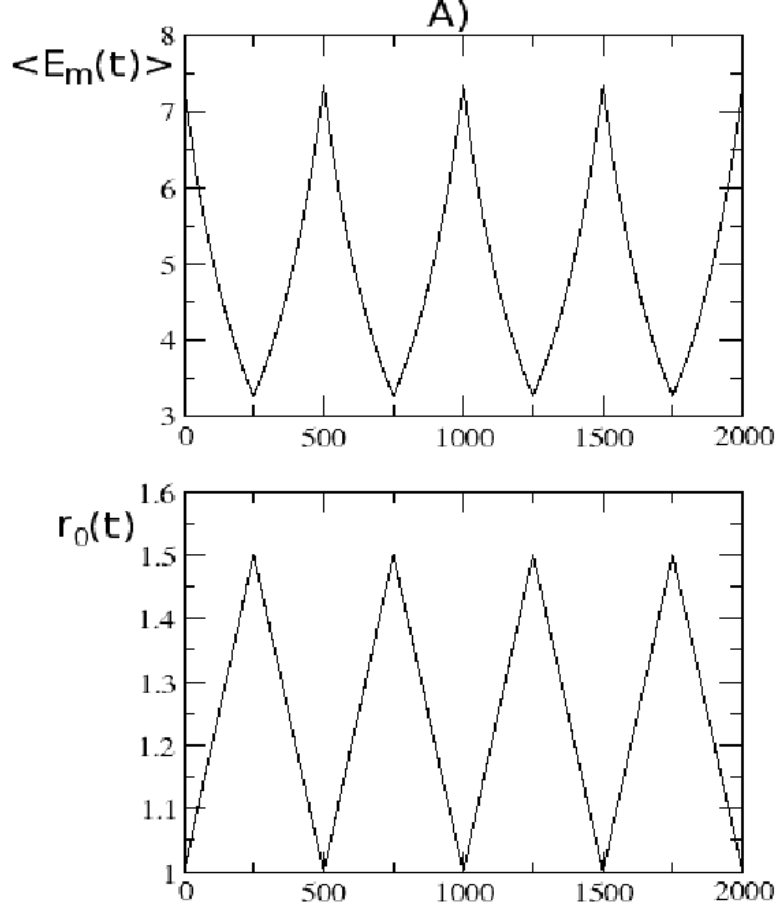

B)
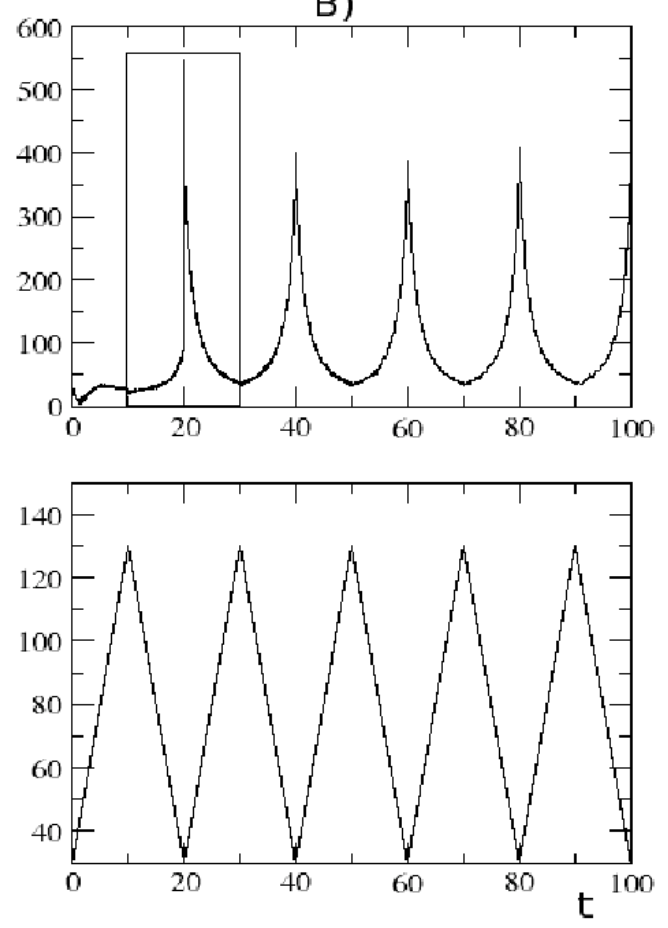

FIG. 4. Time-dependence of the quantum mechanical average energy for the circular billiard with non-harmonically oscillating radius for different oscillation parameters, $\left.m=1, l=1, \mathrm{~A}) \mathrm{T}=500, v=0.002, \rho_{0}=1.0, \mathrm{~B}\right) \mathrm{T}=20$, $v=10, \rho_{0}=30$

Thus, we have derived the full-cycle propagator for the non-harmonically breathing circle. Eq.(38) implies that the frequency of the harmonic oscillator in Eq.(14) has a periodic delta-kicking form. This reduces our problem to solving the Schrödinger equation with a delta-kicking potential (where between the kicks circle expands/contracts linearly), whose solution can be obtained in terms of the full-cycle propagator describing the (exact) evolution of the wave function within one period. Having obtained the full-cycle propagator, we can find solutions for the full time-period $0<t<T$, from which one can construct the solutions for any number of periods. 


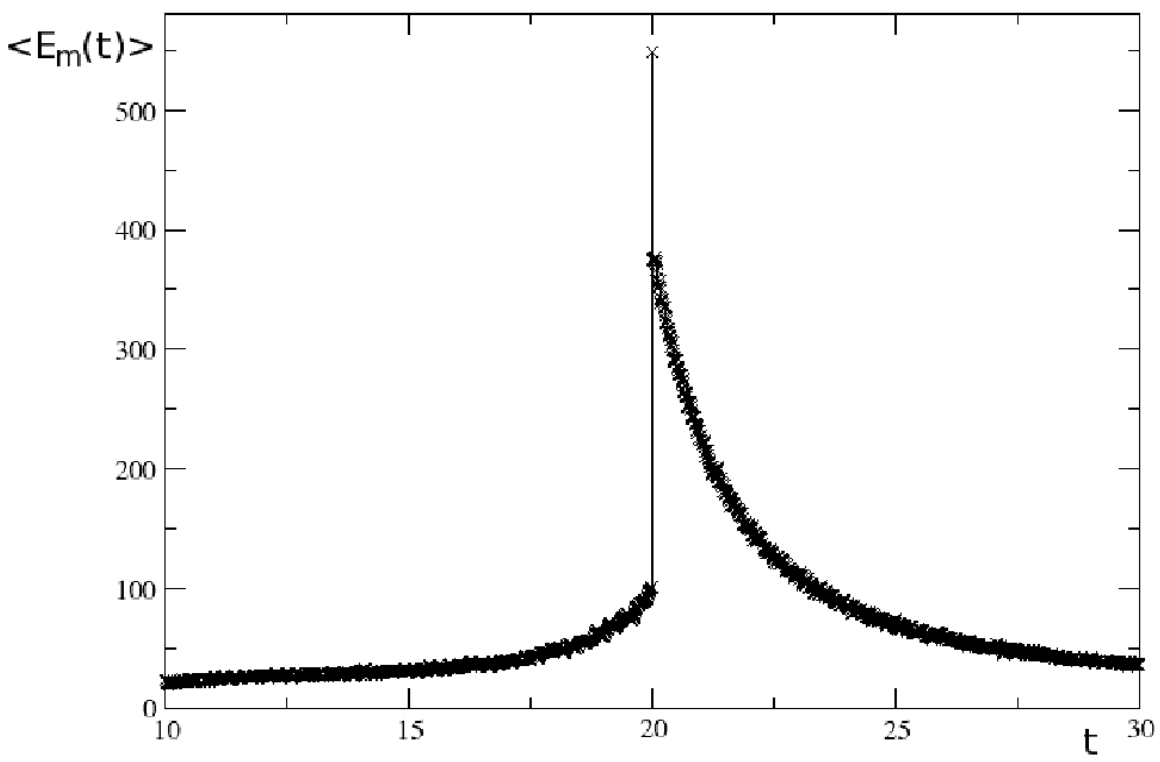

FIG. 5. Magnification of the selected part in Fig.2(B)

In Fig. 4, the time-dependence of the average energy is plotted for different values of $v, T$ and $\rho_{0}$. The expansion coefficients for the initial state (in Eq. (37)) are chosen as:

$$
A_{n}(0)=\int_{0}^{1} \varphi_{n}(y) \varphi_{l}(y) \exp \left(-\frac{i}{2} \dot{r}_{0}(t) r_{0}(0) y^{2}\right) d y
$$

so that $\langle E(0)\rangle=\frac{\lambda_{m l}^{2}}{2 r_{0}^{2}}$.

It is clear that in the adiabatic regime (for high oscillation periods), the timedependence of $\langle E(t)\rangle$ is periodic and for each period it can be separated into symmetric parts, which correspond to linearly expanding and contracting circle average energies (Fig. 4(A)). However, this symmetry is broken in the non-adiabatic regime, corresponding to small oscillation periods. This can be seen from Fig. $4(\mathrm{~B})$, where $\langle E(t)\rangle$ is plotted for $T=20, v=10$ and $\rho_{0}=30$. Such a behavior can be explained by the fact that in the adiabatic regime, the particle follows the wall's motion, while for higher frequencies, it cannot follow the wall's motion. This leads to a breaking of the symmetry, i.e. parts of $\langle E(t)\rangle$ (within one period) corresponding to contracting and expanding circles are not symmetric. This can be clearly seen from Fig. 5, which presents the magnification of a part of the $\langle E(t)\rangle$-curve (Fig. $4(\mathrm{~B})$ ) for the time interval from 10 to 30. It is clear that the maximum value of the left hand part (corresponding to contracting circle) is much smaller than that of the right hand part corresponding to the expanding circle. Such an asymmetry is caused by the presence of a delta-function in the Schrödinger equation. As follows from Eq. (38), the potential in the Schrödinger equation has a jump at $t=T / 2$. This jump is as high as the value of $v$ in Eq. (38). Therefore for smaller velocities the jump is quite small, while for higher values of $v$ it is much larger and can be clearly seen in Fig. 5 .

\section{Harmonically breathing circle}

As we have seen in the previous sections, an analytical solution of the Schrödinger equation of the circular billiard with a time-dependent radius and corresponding boundary conditions is possible in a few cases of the time-dependence only. In particular, no analytical 
solution can be obtained in the case of a harmonic time-dependence, since time and coordinate variables cannot be separated in the Schrödinger equation. In this section, we consider the case of a harmonically oscillating radius which is given by:

$$
r_{0}(t)=a+b \cos (\omega t)
$$

where $\omega$ is the oscillation frequency. The classical dynamics of circular billiards with oscillating boundaries have been investigated in the context of Fermi acceleration [29] and particle motion inside collectively excited nuclei [32]. In Fig. 6, the time-dependence of the energy averaged over an ensemble of 1000 trajectories is plotted for $\omega=2$ and $\omega=5$ for the classical system. The growth of the energy is strongly suppressed for both values of the frequency. It is clear that the character of suppression is the same for both frequencies, although the critical value at which suppression starts is higher for higher frequency values. This is in good accordance with previous studies of the breathing circle, see, e.g., Refs. [29,30].

To numerically solve Eq. (14) with the boundary conditions given by Eq. (42), we expand the function $\phi(y, t)$ in terms of the eigenfunctions of Eq.(14) at $C=0$ :

$$
\phi(y, t)=\sum_{n} c_{n}(t) \varphi_{n}(y)
$$

where:

$$
\varphi_{n}(y)=\frac{\sqrt{2 y} J_{m}\left(\lambda_{m n} y\right)}{J_{m+1}\left(\lambda_{m n}\right)}
$$

Inserting the expansion (43) into Eq.(14) we have:

$$
i r_{0}^{2} \sum_{n} \dot{c}_{n} \varphi_{n}=\sum_{n} c_{n}\left(\frac{\lambda_{m n}^{2}}{2}+\frac{1}{2} r_{0}^{3} \ddot{r}_{0} y^{2}\right) \varphi_{n} .
$$

By multiplying with $\varphi_{k}^{*}$ and integrating over $y$ from 0 to 1 , we get a system of differential equations for the expansion coefficients $c_{n}(t)$ :

$$
\dot{c}_{n}(t)=-\frac{i}{2 r_{0}^{2}} \lambda_{m n}^{2} c_{n}-\frac{i}{2} \ddot{r}_{0} r_{0} \sum_{k} M_{k n} c_{k},
$$

where:

$$
M_{k n}=\int_{0}^{1} \varphi_{n}(y) \varphi_{k}(y) y^{2} d y .
$$

The system of differential equations (46) is solved using ZVODE package [26], which uses variable-coefficient methods. The number of basis functions for the results presented in Figs. (7-10) was taken as 200, while for those in the Fig. 9 we used 3000 basis functions. The convergence of the calculations are checked by increasing the number of basis functions.

Solving Eqs.(46) numerically, we obtain $\phi(y, t)$ and thus the radial wave functions $R(y, t)$. The average energy can be calculated as:

$$
\begin{gathered}
\left\langle E_{m}(t)\right\rangle=\int_{0}^{1} R^{*}(y, t) H R(y, t) y d y= \\
=\frac{1}{2 r_{0}^{2}}\left(\sum_{n}\left|c_{n}\right|^{2} \lambda_{m n}^{2}+r_{0}^{2} \dot{r}_{0}^{2} \int_{0}^{1} y^{2}|\phi|^{2} d y+2 r_{0} \dot{r}_{0} I m \int_{0}^{1} y \phi^{*} \frac{\partial \phi}{\partial y} d y\right) .
\end{gathered}
$$

Using Eq. (47), we numerically compute the average energy as a function of time for different oscillation frequencies. The initial values of the expansion coefficients can be chosen using Eq. (41). Then, for harmonically oscillating boundary (Eq. (42)) the expansion coefficients of the initial state become $c_{n}(0)=\delta_{n l}$, because $\dot{r}_{0}(0)=0$ in Eq. (41). 


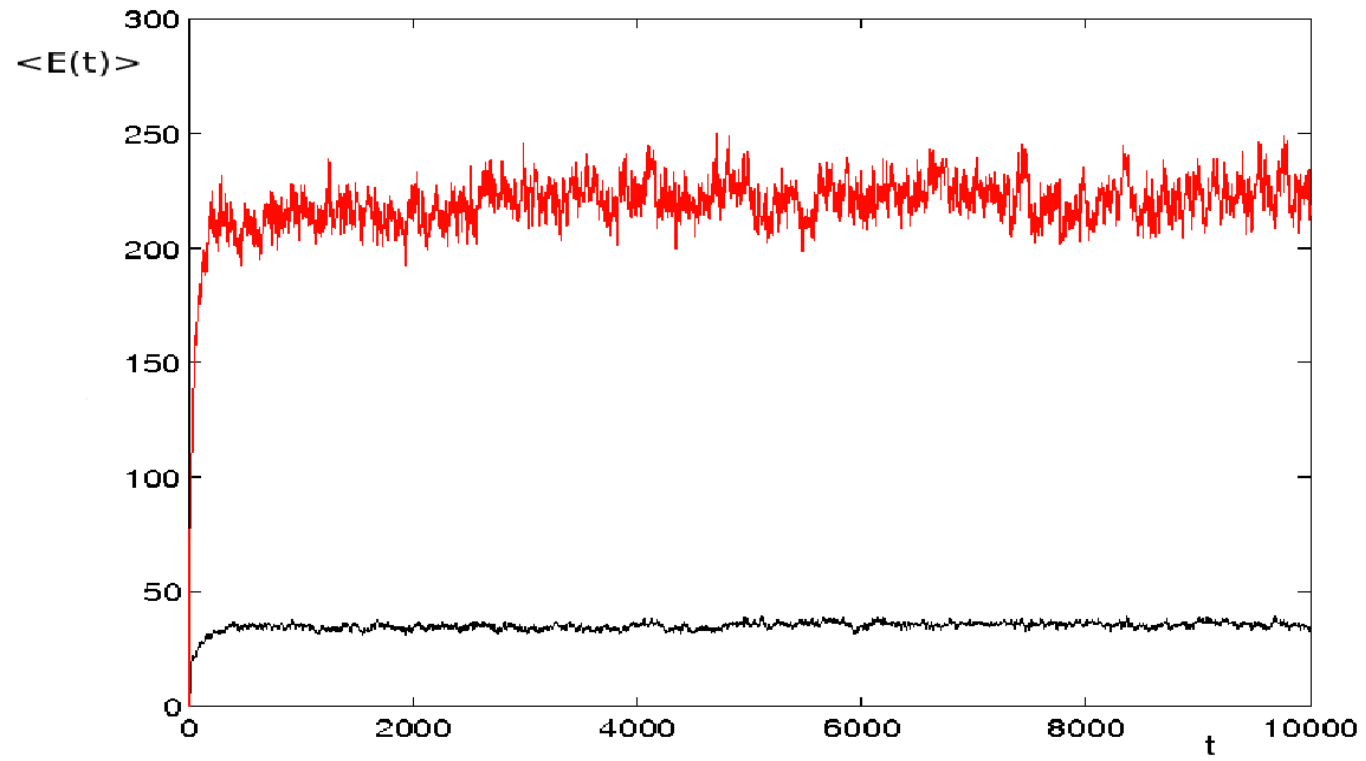

FIG. 6. Time-dependence of the classical average energy for circular billiard with harmonically oscillating radius; black $-r_{0}(t)=20+\cos (2 t)$, red $-r_{0}(t)=$ $20+\cos (5 t)$
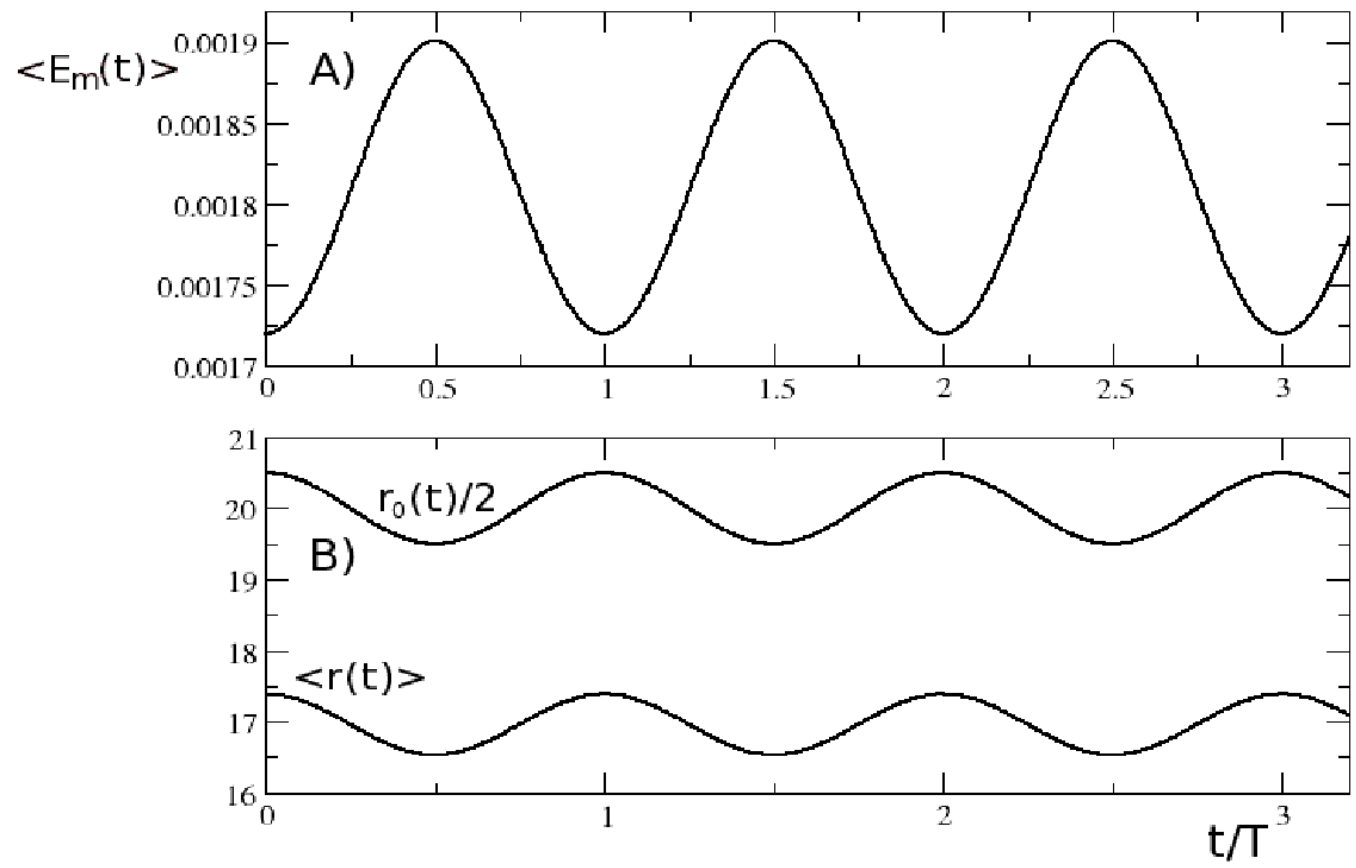

FIG. 7. Time-dependence of the quantum average energy (A) and mean position (B) for the circular billiard with harmonically oscillating radius; $m=0$, $l=1, r_{0}(t)=40+\cos (0.0005 t)$

Furthermore, we compare $\left\langle E_{m}(t)\right\rangle$ for different regimes of the wall's motion: a) slowly oscillating (adiabatic) wall, 


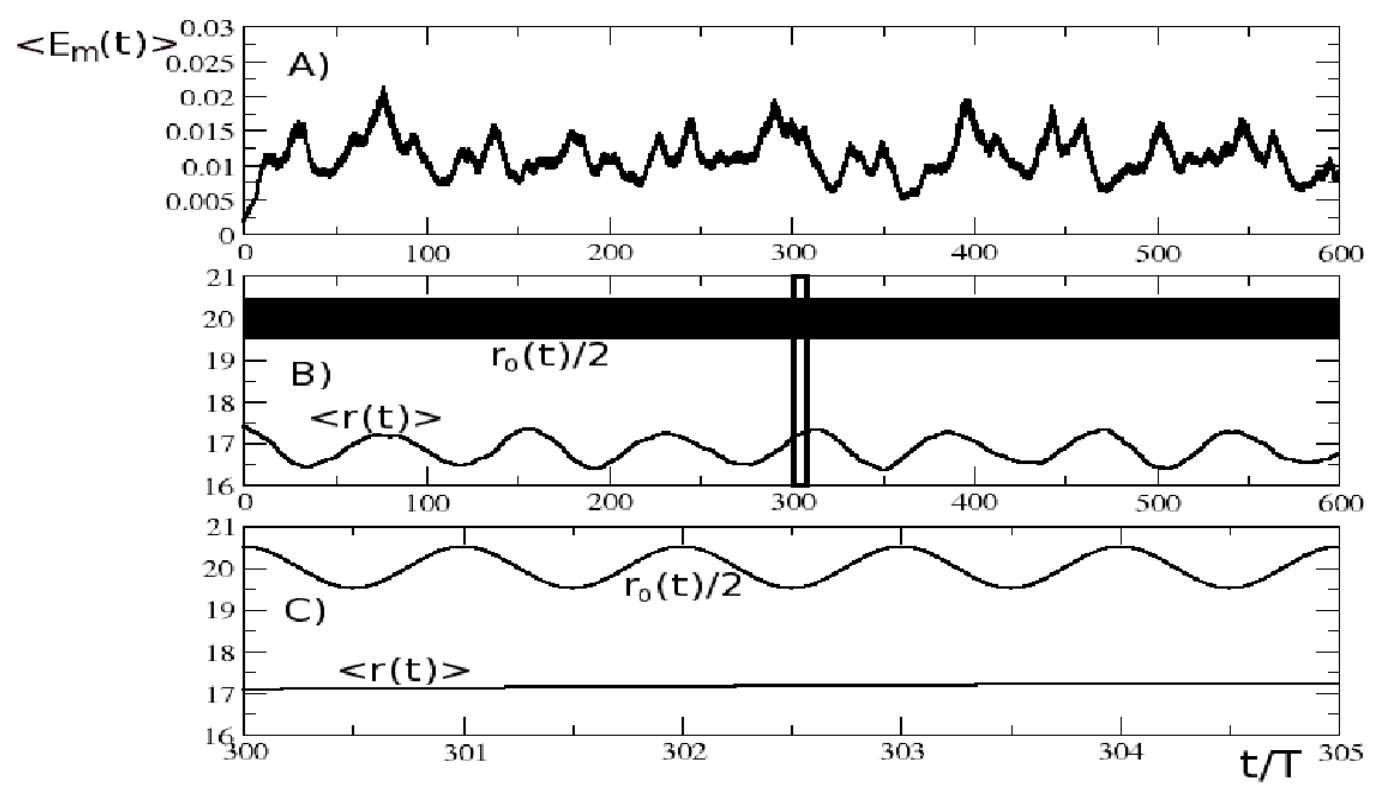

Fig. 8. Time-dependence of the quantum average energy (A) and mean position (B) for the circular billiard with harmonically oscillating radius; $m=0$, $l=1, r_{0}(t)=40+\cos (0.6 t)$. (C) is the magnification of the selected part in (B)

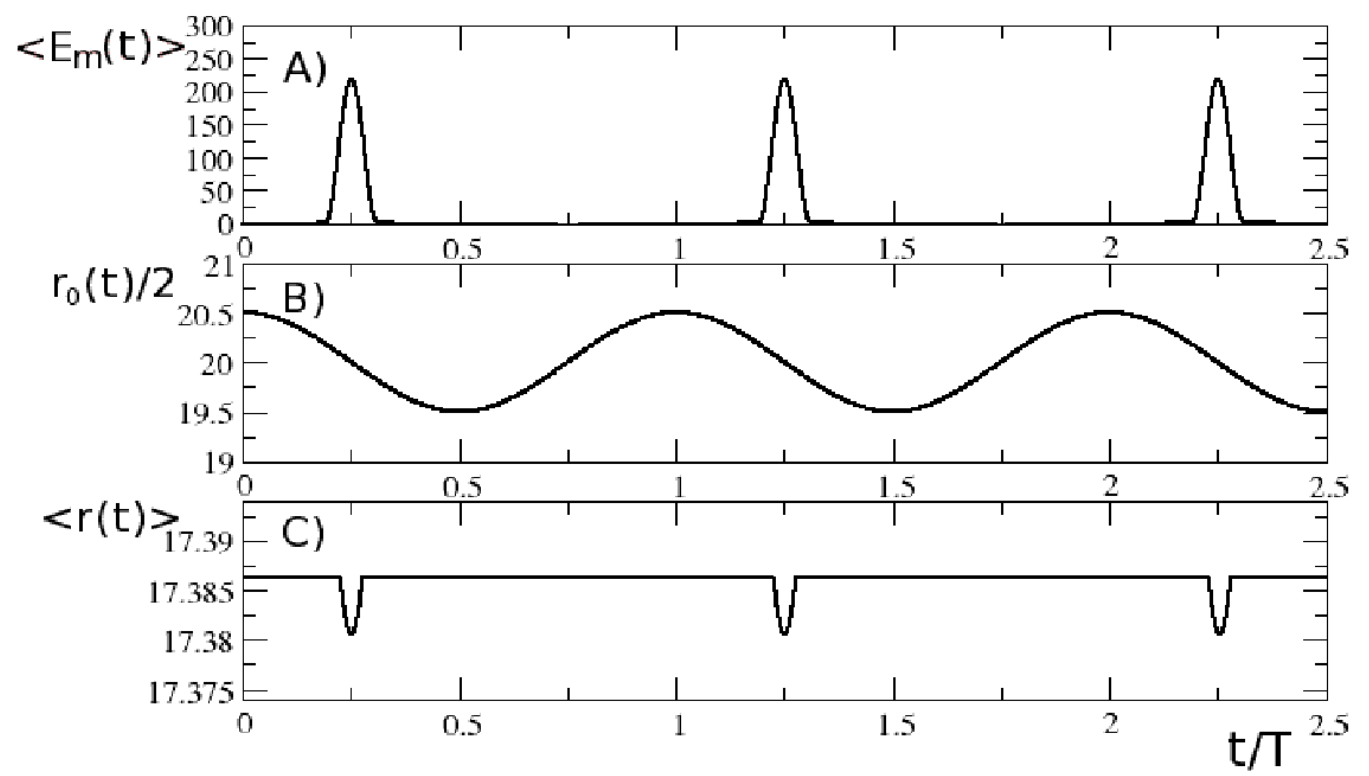

FIG. 9. Time-dependence of the quantum average energy $(\mathrm{A}), r_{0}(t) / 2(\mathrm{~B})$ and mean position $(\mathrm{C})$ for the circular billiard with harmonically oscillating radius; $m=0, l=1, r_{0}(t)=40+\cos (250 t)$

b) high oscillation frequency,

c) intermediate wall oscillation frequencies.

In Fig. $7(\mathrm{~A})$, the time-dependence of the average energy, $\left\langle E_{m}(t)\right\rangle$ is plotted for the adiabatic regime $\left(\omega=5 \times 10^{-4}\right)$. As can be seen from this figure, $\left\langle E_{m}(t)\right\rangle$ is periodic in time 


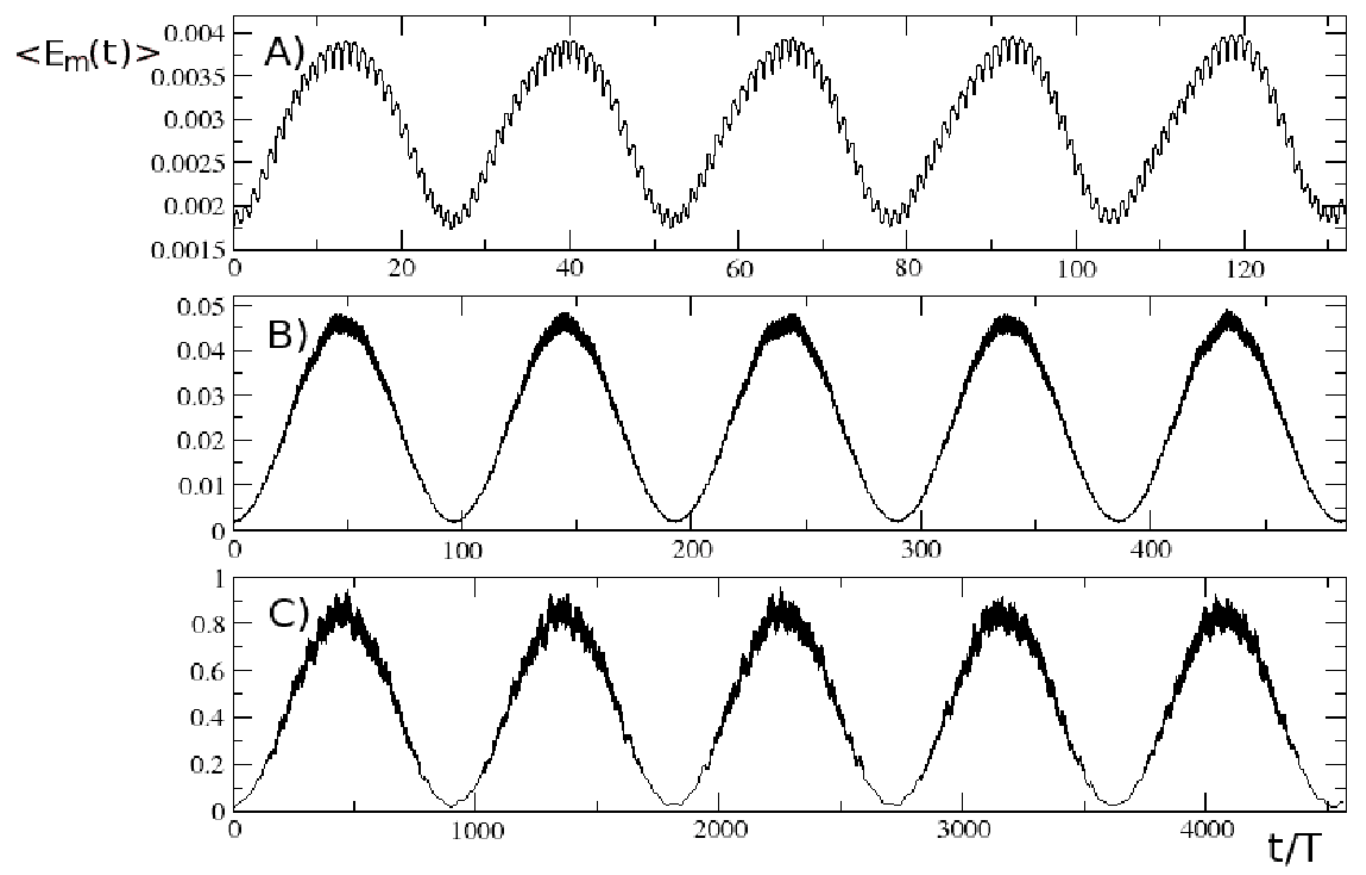

FIG. 10. Time-dependence of the quantum average energy for the circular billiard with harmonically oscillating radius; $m=0, l=1, r_{0}(t)=40+\cos (\omega t)$, A) $\omega=0.02$, B) $\omega=0.1$, C) $\omega=1.7$

and the period is the same as the period of the applied driving law. This can be explained by the fact that in the adiabatic regime, the particle follows the wall's motion. This is clearly seen from Fig. $7(\mathrm{~B})$, where $r_{0}(t) / 2$ is compared with the expectation value of the position of the particle in billiard, $\langle r(t)\rangle$.

Fig. 8(A) presents $\left\langle E_{m}(t)\right\rangle$ for higher values of the wall's oscillation frequency, $(\omega=$ $0.6)$. It is clear that the periodicity of $\left\langle E_{m}(t)\right\rangle$ is broken and this can be explained by Fig. 8(B), where $r_{0}(t) / 2$ and the mean position are compared for this regime. For this value of the frequency, the motion of the billiard particle is no longer adiabatic and it doesn't follow the wall's motion.

In Fig. 9(A), the time-dependence of the average energy is plotted for a very high frequency value, $\omega=250$. It is clear from this plot that the periodicity of $\left\langle E_{m}(t)\right\rangle$ is recovered in this highly oscillating regime. Comparison of $\langle r(t)\rangle$ and $r_{0}(t) / 2$ in Fig. 9(B) shows that the particle doesn't "feel" the wall's motion in this regime.

Finally, a remarkable feature of the harmonically breathing circle can be observed in the intermediate regime. Namely, for some values of the frequency $\left\langle E_{m}(t)\right\rangle$ can be timeperiodic with the period which is much larger than that of wall's oscillation. Fig. 10 presents the plots of the average energy for $(\omega=0.02, \omega=0.1, \omega=1.7)$ which exhibit such a periodicity. The appearance of such periodicity for intermediate oscillation frequencies may be caused by the existence of a special resonance-like regime of motion where the periodic motion is possible for certain frequencies only.

To qualitatively determine the border between the adiabatic and non-adiabatic regimes, we study the behavior of the expansion coefficients, $c_{n}$ for different oscillation frequencies. It is clear that billiard wall's position changes very slowly, the wavefunction of the billiard particle should be slightly different than that of the static billiard. Therefore, in the wavefunction of the adiabatically expanding (contracting) billiard, which is given by Eq.(43) only 

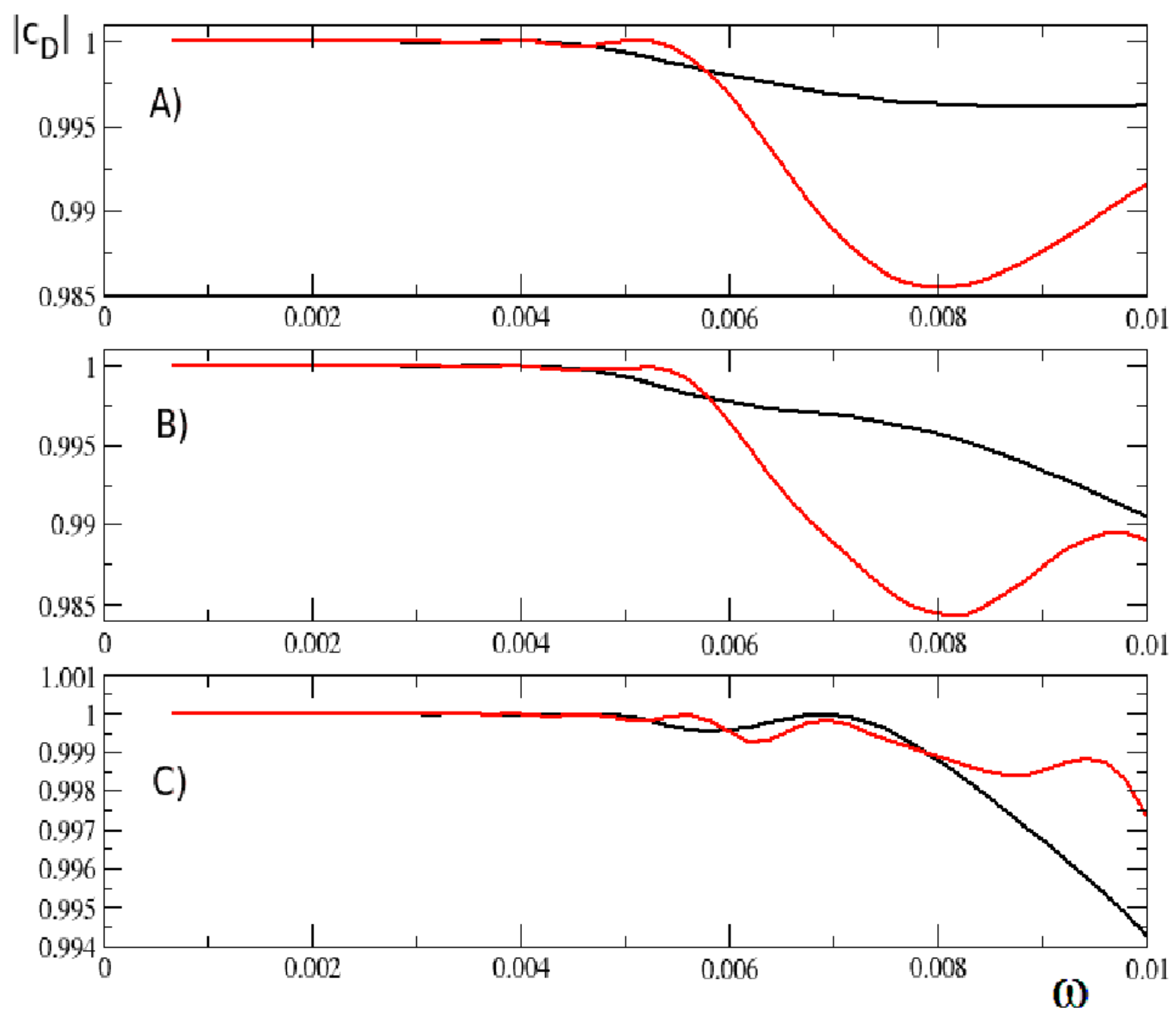

FIG. 11. The absolute value of the dominating expansion coefficient $\left(\left|c_{D}\right|\right)$ as a function of $\omega$, measured after one period (black curves), and after two periods (red curves). Initial states are A) $m=0, n=1, \mathrm{~B}) m=0, n=2, \mathrm{C}$ ) $m=0, n=3$

one expansion coefficient, $c_{D}$ (which corresponds to the initial state) is dominating. Thus, the dependence of this coefficient on the expansion (contraction) rate can be considered as an indicator for the fact whether wall's motion adiabatic or not. In application to the harmonically breathing billiard, this implies that the breaking of the dominance of the coefficient $c_{D}$ coefficient when the oscillation frequency reaches some threshold value can be considered as a breaking of the adiabatic regime. Fig. 11 represents $\left|c_{D}\right|$ as a function of the wall's oscillation frequency, $\omega$. Up to a certain value of $\omega,\left|c_{D}\right|$ is approximately equal to 1 . After exceeding some (critical) value of $\omega,\left|c_{D}\right|$ starts to become less than 1, which implies breaking of the adiabatic regime.

Thus, we can conclude that for the harmonically breathing circle the behavior of the average energy as a function of time is mostly similar to that of harmonically oscillating $1 D$ box, studied in the Ref. [28]. However, unlike to that system, in the breathing circle, $\left\langle E_{m}(t)\right\rangle$ 
can be time-periodic even for certain frequency values, which belong to the intermediate frequency range.

\section{Conclusions}

We have studied the quantum dynamics of a circular billiard with different driving laws for the radius. An exact analytical solution was obtained for cases of monotonically expanding and contracting circles. Non-harmonically time-periodic boundary conditions (sawtooth-like motion) were considered in terms of the analytically-derived full-cycle propagator. Using this propagator, the time-dependence of the average energy was calculated. It was found that in the adiabatic regime, when the wall moves slowly, the time-dependence of the average energy was periodic and for each period, $\langle E(t)\rangle$ can be constructed from corresponding $\langle E(t)\rangle_{\mathrm{s}}$ of linearly expanding and contracting circles. In other words, the part of the curve for the average energy corresponding to one period consisted of two symmetric parts, describing linearly expanding and contracting circles. However, for smaller oscillation periods when adiabaticity was broken, such symmetry was broken, though $\langle E(t)\rangle$ was still periodic. To explore more deeply the difference between adiabatic and highly adiabatic regimes, we considered the case when billiard wall suddenly disappears. It was found for this case that the mean position of the billiard particle grew monotonically in time, which implies that over a long period, the motion becomes infinite.

The case of the harmonically breathing circle was studied by solving the timedependent Schrödinger equation numerically by means of a basis set expansion. When the oscillation frequency of the radius was very small (compared to the initial frequency, $\omega_{0}$ ), the systems remained in the adiabatic regime and $\langle E(t)\rangle$ was periodic with the same period as that of the applied driving law. Such periodicity broken down by increasing the oscillation frequency. However, for some intermediate range frequencies, $\langle E(t)\rangle$ can become periodic in time with a period much larger than that of the driving law. For very high oscillation frequencies, the average energy became time-periodic again, with the same period as that of the driving law. The border between adiabatic and non-adiabatic regime is roughly defined by the dependence of the dominating expansion coefficient in Eq.(43) on the wall's oscillation frequency. Namely, the situation when the dominance of these coefficients breaks can be considered as a fingerprint for the transition from an adiabatic into a non-adiabatic regime. In our opinion, the explanation of such periodicity requires deeper exploration of the studied system, which will be the subject of forthcoming research. Finally, the importance of the above study is due to its direct relevance to quantum Fermi acceleration in confined geometries and the problem of quantum dynamics in driven systems. The latter is of importance for many mesoscopic and nanoscale systems such as quantum dots, confined cold atoms and molecules. Finally, we note that extension of the above study to the case of open quantum billiards is of importance because of their direct relevance to quantum dots. Currently, such studies are ongoing.

\section{Acknowledgement}

This work is supported in part by a grant of Volkswagen Foundation ( Nr. I/82 136). 


\section{References}

[1] Stöckmann H.-J. Quantum Chaos: An Introduction. Cambridge University Press, Cambridge, UK (1999).

[2] Eckhardt B. Quantum mechanics of classically non-integrable systems. Phys. Rep., 163, P. 205-297 (1988).

[3] Gutzwiller M.C. Chaos in Classical and Quantum Mechanics. Springer, New York (1990).

[4] Loskutov A.Yu., Ryabov A.B. and Akinshin L.G. Properties of some chaotic billiards with timedependent boundaries. J. Phys. A, 33, P. 7973 (2000).

[5] Loskutov A.Yu. and Ryabov A.B. Particle Dynamics in Time-Dependent Stadium-Like Billiards. $J$. Stat. Phys., 108, P. 995-1014 (2002).

[6] De Carvalho R.E., De Souza F.C. and Leonel E.D. Fermi acceleration on the annular billiard: a simplified version, J. Phys. A: Math. Gen., 39, P. 3561(2006).

[7] Lenz F., Diakonos F.K. and Schmelcher P. Classical dynamics of the time-dependent elliptical billiard. Phys. Rev. E, 76, P. 066213 (2007).

[8] Lenz F., Diakonos F.K. and Schmelcher P. Tunable Fermi Acceleration in the Driven Elliptical Billiard. Phys. Rev. Lett., 100, P. 014103 (2008).

[9] Cohen D., Wisniacki D.A. Stadium billiard with moving walls. Phys. Rev. E, 67, P. 026206 (2003).

[10] Doescher S.W. and Rice M.H. Infinite Square-Well Potential with a Moving Wall. Am. J. Phys., 37, P. 1246 (1969).

[11] Munier A., Burgan J.R., Feix M. and Fijalkow E. Schrödinger equation with time-dependent boundary conditions. J. Math. Phys., 22, P. 1219 (1981).

[12] Pinder D.N. The contracting square quantum well. Am. J. Phys., 58, P. 54 (1990).

[13] Razavy M. Time-dependent harmonic oscillator confined in a box. Phys. Rev. A, 44, P. 2384 (1991).

[14] Pereshogin P., Pronin P. Effective Hamiltonian and Berry phase in a quantum mechanical system with time dependent boundary conditions. Phys. Lett. A, 156, P. 12-16 (1991).

[15] Scheininger C. and Kleber M. Quantum to classical correspondence for the Fermi-acceleration model. Physica D, 50, P. 391-404 (1991).

[16] Makowski A.J. and Dembinski S.T. Exactly solvable models with time-dependent boundary conditions. Phys. Lett. A, 154, P. 217-220 (1991).

[17] Makowski A.J. and Peplowski P. On the behaviour of quantum systems with time-dependent boundary conditions. Phys. Lett. A, 163, P. 143-151 (1992).

[18] Makowski A.J. Two classes of exactly solvable quantum models with moving boundaries. J. Phys. A: Math. Gen., 25, P. 3419 (1992).

[19] Willemsen J.E. Exact solution of the wave dynamics of a particle bouncing chaotically on a periodically oscillating wall. Phys. Rev. E, 50, P. 3116 (1994).

[20] Morales D.A., Parra Z., Almeida R. On the solution of the Schrödinger equation with time dependent boundary conditions. Phys. Lett. A, 185, P. 273-276 (1994).

[21] C. Yuce. Singular potentials and moving boundaries in 3D. Phys. Lett. A, 321, P. 291-294 (2004).

[22] Jose J.V., Gordery R. Study of a quantum fermi-acceleration model. Phys. Rev. Lett., 56, P. 290 (1986).

[23] Karner G. On the quantum Fermi accelerator and its relevance to 'quantum chaos'. Lett. Math. Phys. A, 17, P. 329-339 (1989).

[24] Seba P. Quantum chaos in the Fermi-accelerator model. Phys. Rev. A, 41, P. 2306 (1990).

[25] Jana T.K., Roy P. A class of exactly solvable Schrödinger equation with moving boundary condition. Phys. Lett. A, 372, P. 2368-2373 (2008).

[26] www.netlib.org

[27] Godoy S. Diffraction in time: Fraunhofer and Fresnel dispersion by a slit. Phys. Rev. A, 65, P. 042111 (2002).

[28] Glasser M.L., Mateo J., Negro J. and Nieto L.M. Quantum infinite square well with an oscillating wall. Chaos, Solitons and Fractals, 41, P. 2067-2074 (2009).

[29] Koiller J., Markarian R., Kamphorst S.O. and De Carvalho S.P. Time-dependent billiards. Nonlinearity, 8, P. 983 (1995).

[30] Kamphorst S.O., De Carvalho S.P. Bounded gain of energy on the breathing circle billiard. Nonlinearity, 12, P. 1363-1371 (1999).

[31] Badrinarayanan R., Jose J.V., Chu G. Quantum manifestations of classical chaos in a Fermi accelerating disk. Physica D, 83, P. 1-29 (1995). 
[32] Burgio G.F., Baldo M., Rapisarda A., Schuck P. Chaoticity in vibrating nuclear billiards. Phys. Rev. C, 52, P. 2475 (1995).

[33] Robinett R.W., Heppelmann S. Quantum wave-packet revivals in circular billiards. Phys. Rev. A, 65, P. 062103 (2002).

[34] Robinett R.W. Quantum mechanics of the two-dimensional circular billiard plus baffle system and half-integral angular momentum. Eur. J. Phys., 24, P. 231 (2003).

[35] Abramowitz M., Stegun I.A. Handbook of mathematical functions. M.: Nauka. (1964). 